\title{
Threshold Dynamics of a Vector-Borne Epidemic Model for Huanglongbing with Impulsive Control
}

\author{
Jianping Wang*, Feifei Feng, Zhicai Guo, Hengmin Lv, Juanjuan Wang \\ The Department of Basic Course Education, Ji'an College, Ji'an, China \\ Email: *jpwmath@126.com
}

How to cite this paper: Wang, J.P., Feng, F.F., Guo, Z.C., Lv, H.M. and Wang, J.J. (2019) Threshold Dynamics of a Vector-Borne Epidemic Model for Huanglongbing with Impulsive Control. Applied Mathematics, 10, 196-211.

https://doi.org/10.4236/am.2019.104015

Received: March 25, 2019

Accepted: April 21, 2019

Published: April 24, 2019

Copyright $\odot 2019$ by author(s) and Scientific Research Publishing Inc. This work is licensed under the Creative Commons Attribution International License (CC BY 4.0).

http://creativecommons.org/licenses/by/4.0/

\begin{abstract}
In this paper, the basic reproduction number is calculated for Huanglongbing (HLB) model with impulses which is a vector-borne epidemic model with impulses. For controlling HLB, farmers' experience is replanting of healthy plants and removing infected plants. To reflect the real world, we construct an impulsive control model which considers replanting of healthy plants and removing infected plants at one fixed time. By analyzing the model, we conclude that the disease-free equilibrium is globally asymptotically stable if the basic reproduction number $R_{0}<1$, and we prove that the HLB is permanence if the basic reproduction number $R_{0}>1$.
\end{abstract}

\section{Keywords}

Huanglongbing, Impulsive Control, Basic Reproduction Number, Disease-Free Periodic Solution, Permanence

\section{Introduction}

Huanglongbing (HLB) is one of the most serious problems of citrus worldwide which caused by the bacteria Candidatus Liberibacter spp., whose name in Chinese means "yellow dragon disease", was first reported from southern China in 1919 and is now known to occur in next to 40 different Asian, African, Oceanian, South and North American countries [1]. HLB has no cure and affects all citrus varieties, reducing the productivity of orchards because the fruits of infected plants have poor quality and, in extreme cases, infection leads to plant death [2]. HLB symptoms are virtually the same wherever the disease occurs. Infected trees show a blotchy mottle condition of the leaves that result in the development of yellow shoots, the early and very characteristic symptom of the disease [1]. As 
we all know, HLB can be spread efficiently by vector psyllids to all commercial cultivars of citrus [3] [4].

Mathematical models play an important role in understanding the epidemiology of vector-transmitted plant diseases. Applications of mathematical approach to plant epidemics were reviewed by Van der Plank [5] and Kranz [6]. There are many authors establish continuous mathematical models to describe the transmission of HLB. Chiyaka et al. [7] proposed a compartmental model of ordinary differential equations for the HLB transmission dynamics within a citrus tree considering 10 dimensions. In [2], Raphael et al. constructed a 6 dimensions model of ordinary differential equations with delay time. However the dynamic behaviors of these models are studied only by using computer simulations.

But, in our real world, farmers' experiences have led to development of integrated management concepts for virus diseases that combine available host resistance, cultural, chemical and biological control measures. A cultural control strategy including replanting, and/or removing (rouging) diseased plants is a widely accepted treatment for plant epidemics which involves periodic inspections followed by removal of the detected infected plants [8] [9] [10] [11] [12]. Periodic replanting of healthy plants or removing (rouging) infected plants in plant-virus disease epidemics is widely used to minimize losses and maximize returns [12]. There are only a few countries have been able to control Asian HLB. São Paulo State (SPS) might be one of the first to be successful. In SPS, encouraging results have been obtained in the control of HLB by tree removal and insecticide treatments against psyllids [13]. Monocrotophos has a short residual effect on psyllid, repeated application is often required to suppress psyllid, which can cause pesticide resistance. Pesticides pollution is also recognized as a major health hazard to human beings and beneficial insects. To deal with these questions, we propose model dealing in detail with the killing efficiency rate and decay rate of pesticides. The residual effects of pesticides (i.e. killing efficiency rate and decay rate) on the threshold conditions are also addressed.

A model for the temporal spread of an epidemic in a closed plant population with periodic removals of infected plants has been considered by Fishman et al. [8]. Integrated management has been found to be more effective at eliminating epidemics. In this paper, according to the above biological background, we develop a hybrid impulsive control model, in which replanting of healthy plants and removing infected plants at one fixed moment and pesticide spraying at another fixed moment are considered, to propose optimal control strategy.

The paper is organized as follows. In Section 2, we formulate the impulsive epidemic model and also simplify the original system (2.1). In Section 3, we introduce some useful lemmas and the basic reproduction number of the model. In Sections 4 and 5, we proved the global stability of the disease-free equilibrium and permanence of the model, respectively. In the finally section, a brief discussion is given. 


\section{Model Formulation}

Let $S_{h}(t), I_{h}(t)$ denote susceptible citrus host and infected citrus host, respectively, and $S_{v}(t), I_{v}(t)$ represent susceptible psyllid and infected psyllid, respectively. We give the following system:

$$
\left\{\begin{array}{l}
\frac{\mathrm{d} S_{h}(t)}{\mathrm{d} t}=-\lambda S_{h}(t) I_{v}(t)-d_{1} S_{h}(t), \\
\frac{\mathrm{d} I_{h}(t)}{\mathrm{d} t}=\lambda S_{h}(t) I_{v}(t)-d_{1} I_{h}(t)-v I_{h}(t), \\
\frac{\mathrm{d} S_{v}(t)}{\mathrm{d} t}=\Lambda-\beta S_{v}(t) I_{h}(t)-d_{2} S_{v}(t), \\
\frac{\mathrm{d} I_{v}(t)}{\mathrm{d} t}=\beta S_{v}(t) I_{h}(t)-d_{2} I_{v}(t), \\
S_{h}\left(t^{+}\right)=S_{h}(t)+\delta, \\
I_{h}\left(t^{+}\right)=(1-\phi) I_{h}(t), \\
S_{v}\left(t^{+}\right)=S_{v}(t), \\
I_{v}\left(t^{+}\right)=I_{v}(t),
\end{array}\right\} \quad t \neq k T, k \in N,
$$

with initial condition

$$
S_{h}\left(0^{+}\right)>0, I_{h}\left(0^{+}\right)>0, S_{v}\left(0^{+}\right)>0, I_{v}\left(0^{+}\right)>0 .
$$

The model is satisfied with the following assumptions.

- $S_{h}, I_{h}, S_{v}$ and $I_{v}$ are left continuous, that is, $S_{h}(t)=S_{h}\left(t^{-}\right)$, $I_{h}(t)=I_{h}\left(t^{-}\right), \quad S_{v}(t)=S_{v}\left(t^{-}\right)$and $I_{v}(t)=I_{v}\left(t^{-}\right)$for all $t \geq 0$.

- $\lambda \geq 0$ is the infected rate of citrus host. $d_{1}>0, v>0$ are the nature death rate and disease induced death rate of citrus, respectively.

- $\Lambda>0$ is constant recruitment rate of psyllid.

- $\beta \geq 0, d_{2}>0$ are the infected rate and nature death rate of psyllid, respectively.

- $\delta \geq 0,0<\phi<1$ are the recruitment rate of citrus and removing rate of infected citrus by impulses, respectively.

- $T>0$ is the interpulse time, i.e., the time between two consecutive pulse replanting and removing.

The following lemma is obvious.

Lemma 2.1. If $S_{h}\left(0^{+}\right)>0, I_{h}\left(0^{+}\right)>0, S_{v}\left(0^{+}\right)>0$ and $I_{v}\left(0^{+}\right)>0$, then $S_{h}(t)>0, I_{h}(t)>0, S_{v}(t)>0$ and $I_{v}(t)>0$ for every $t>0$.

Denote $G:=\left\{\left(S_{h}, I_{h}, S_{v}, I_{v}\right) \in R_{+}^{4} \mid 0 \leq S_{h}+I_{h} \leq N_{h}^{*}, 0 \leq S_{v}+I_{v} \leq N_{v}^{*}\right\}$, where $N_{h}^{*}=\frac{\delta}{1-\mathrm{e}^{-d_{1} T}}, \quad N_{v}^{*}=\frac{\Lambda}{d_{2}}$.

Theorem 2.1. The solutions of system (2.1) with initial condition (2.2) eventually enter into $G$ and $G$ is positively invariant for system (2.1).

Proof: Let $N_{h}=S_{h}+I_{h}, N_{v}=S_{v}+I_{v}$. By system (2.1), we have 


$$
\left\{\begin{array}{l}
\frac{\mathrm{d} N_{h}(t)}{\mathrm{d} t}=-d_{1} N_{h}(t)-v I_{h}(t), \\
\frac{\mathrm{d} N_{v}(t)}{\mathrm{d} t}=\Lambda-d_{2} N_{v}(t), \\
N_{h}\left(t^{+}\right)=N_{h}(t)-\phi I_{h}(t)+\delta, \\
N_{v}\left(t^{+}\right)=N_{v}(t),
\end{array}\right\} t \neq k T,
$$

By the first and third equations of (2.3), we get

$$
\left\{\begin{array}{l}
\frac{\mathrm{d} N_{h}(t)}{\mathrm{d} t} \leq-d_{1} N_{h}, \quad t \neq k T, \\
N_{h}\left(t^{+}\right) \leq N_{h}(t)+\delta, \quad t=k T,
\end{array}\right.
$$

Thus, we have $N_{h} \leq \frac{\delta}{1-\mathrm{e}^{-d_{1} T}}$.

From the second and fourth equations of (2.3), we have

$$
\frac{\mathrm{d} N_{v}(t)}{\mathrm{d} t} \leq \Lambda-d_{2} N_{v}
$$

Then, we have $N_{v} \leq \frac{\Lambda}{d_{2}}$.

Then, from the above analysis, which implies that $G$ is positively invariant.

\section{The Basic Reproduction Number of (2.1)}

Let $\left(R^{n}, R_{+}^{n}\right)$ be the standard ordered $n$-dimensional Euclidean space with a norm $\|\cdot\|$. For $u, v \in R^{n}$, we write $u \geq v$ if $u-v \in R_{+}^{n}, u>v$ if $u-v \in R_{+}^{n} \backslash\{0\}, u \gg v$ if $u-v \in \operatorname{Int}\left(R_{+}^{n}\right)$, respectively.

Set $A(t)$ be cooperative, irreducible and periodic $n \times n$ matrix function with period $\omega(>0), P$ be a $n \times n$ constant matrix, $T$ be a pulse period satisfying $\omega / T=q, q \in N$. Then $\Phi_{A(\cdot)}(t)$ is the fundamental solution matrix of the linear differential equation

$$
\frac{\mathrm{d} y(t)}{\mathrm{d} t}=A(t) y,
$$

and $r\left(P^{q} \Phi_{A(.)}(\omega)\right)$ is the spectral radius of $P^{q} \Phi_{A(\cdot)}(\omega)$. By Perron-Frobenius theorem, $r\left(P^{q} \Phi_{A(.)}(\omega)\right)$ is the principal eigenvalue of $\Phi_{A(.)}(\omega)$ in the sense that it is simple and admits an eigenvector $v^{*} \gg 0$.

Firstly, we introduce some lemmas which will be useful for our further arguments.

Lemma 3.1. [14] Let $\mu=\frac{1}{\omega} \ln r\left(P^{q} \Phi_{A(.)}(\omega)\right)$. Then there exists a positive, $\omega$-periodic function $v(t)$ such that $\mathrm{e}^{\mu t} v(t)$ is a solution of

$$
\left\{\begin{array}{l}
\frac{\mathrm{d} y(t)}{\mathrm{d} t}=A(t) y, \quad t \neq k T, k \in N, \\
y\left(t^{+}\right)=P y(t), \quad t=k T, k \in N .
\end{array}\right.
$$

In what follows, we give the basic reproduction number $R_{0}$ for system (2.1). 
Similar to Yang and Xiao [15].

An impulsive periodic differential mathematical model in which impulses occur at fixed times may be described as follows:

$$
\left\{\begin{array}{l}
\frac{\mathrm{d} x(t)}{\mathrm{d} t}=f(t, x), t \neq k T, \\
x\left(t^{+}\right)=x(t)+I(t), t=k T,
\end{array}\right.
$$

where $f: R^{+} \times \Omega \rightarrow R^{k}$ is $\omega$-periodic function and $q=\omega / T$, for $t=k t$, $x\left(k t^{+}\right)=\lim _{x} x(k t+h), k \in N, N=1,2, \cdots$ and $\Omega$ is an open set.

Let $\mathcal{F}_{i}\left(t, t^{0}, x(t)\right)$ be the input rate of newly infected individuals in the $i$-th compartment, and $\mathcal{V}_{i}(t, x(t))=\mathcal{V}_{i}^{-}(t, x(t))-\mathcal{V}_{i}^{+}(t, x(t))$ where $\mathcal{V}_{i}^{+}(t, x(t))$ be the input rate of individuals by other means, and $\mathcal{V}_{i}^{-}(t, x(t))$ be the rate of transfer of individuals out of compartment $i$; then $\mathcal{V}_{i}(t, x(t))$ denotes the net transfer rate out of compartments. We suppose that $x\left(t_{k}\right)$ immediately after pulses equals

$$
x\left(k t^{+}\right)=x(k t)+I(k t)=\psi(x(k t)),
$$

where $\psi: \Omega \rightarrow \Omega, \Omega \in R^{n}, \psi \in C^{1}(\Omega, \Omega)$.

Denote

$$
\begin{gathered}
x=\left(x_{1}, x_{2}, \cdots, x_{n}\right)^{\mathrm{T}}, \\
\mathcal{F}(t, x)=\left(\mathcal{F}_{1}(t, x), \mathcal{F}_{2}(t, x), \cdots, \mathcal{F}_{n}(t, x)\right)^{\mathrm{T}}, \\
\mathcal{V}(t, x)=\left(\mathcal{V}_{1}(t, x), \mathcal{V}_{2}(t, x), \cdots, \mathcal{V}_{n}(t, x)\right)^{\mathrm{T}},
\end{gathered}
$$

where $A^{\mathrm{T}}$ denotes the transpose of $A$, and $x_{1}, x_{2}, \cdots, x_{n}$ are $n$ homogeneous compartments in a heterogeneous population, with each $x_{i} \geq 0$ being the number of individuals in each compartment. Assume that the compartments sort by two types, with the first $m$ compartments $x_{1}, x_{2}, \cdots, x_{m}$ the infected individual, and $x_{m+1}, \cdots, x_{n}$ the uninfected individuals. Denote

$$
\begin{gathered}
X=\left(x_{1}, \cdots, x_{m}\right), Y=\left(x_{m+1}, \cdots, x_{n}\right), \\
\psi=(h, g)^{\mathrm{T}}, h=\left(\psi_{1}, \cdots, \psi_{m}\right), g=\left(\psi_{m+1}, \cdots, \psi_{n}\right), \\
h \in C^{1}\left(\Omega, R^{m}\right), g \in C^{1}\left(\Omega, R^{n-m}\right) .
\end{gathered}
$$

Now, system (3.2) can be written as

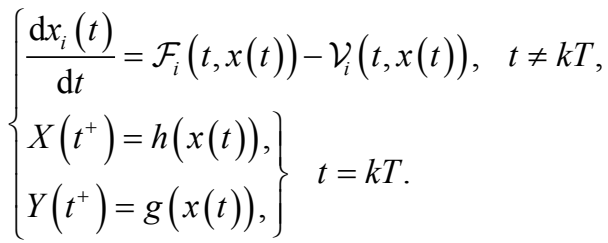

Define $X_{s}$ to be the set of all disease-free states:

$$
X_{s}=\left\{x \geq 0 \mid x_{i}=0, i=1, \cdots, m\right\} .
$$

Furthermore, assume that

$$
\tilde{x}(t)=\left(0, \cdots, 0, \tilde{x}_{m+1}(t), \cdots, \tilde{x}_{n}(t)\right)^{\mathrm{T}}
$$


be a disease-free periodic solution over the $k$-th time interval $(k T,(k+1) T]$ with $\tilde{x}_{i}(t)>0, m+1 \leq i \leq n$, for all $t \geq 0$.

Let

$$
\begin{gathered}
F(t)=\left(\frac{\partial \mathcal{F}_{i}(t, \tilde{x}(t))}{\partial x_{j}}\right)_{1 \leq i, j \leq m}, \quad V(t)=\left(\frac{\partial \mathcal{V}_{i}(t, \tilde{x}(t))}{\partial x_{j}}\right)_{1 \leq i, j \leq m}, \\
M(t)=\left(\frac{\partial f_{i}(t, \tilde{x}(t))}{\partial x_{j}}\right)_{m+1 \leq i, j \leq n}, \quad Q=\left(\frac{\partial \psi_{i}(t, \tilde{x}(t))}{\partial x_{j}}\right)_{m+1 \leq i, j \leq n},
\end{gathered}
$$

and $P=\left(\frac{\partial \psi_{i}(t, \tilde{x}(t))}{\partial x_{j}}\right)_{1 \leq i, j \leq m}$, where $\mathcal{F}_{i}(t, x(t)), \mathcal{V}_{i}(t, x(t)), \quad f_{i}(x(t)), x_{i}$ and $\psi_{i}$ are the $i$-th component of $\mathcal{F}(t, x(t)), \mathcal{V}(t, x(t)), f(t, x(t)), x$ and $\psi$, respectively.

We make the following assumptions, which are the same biological meanings as those by Wang and Zhao [16] and Yang and Xiao [15].

(H1) If $x \geq 0$, then $\mathcal{F}_{i}, \mathcal{V}_{i}^{+}, \mathcal{V}_{i}^{-} \geq 0$ for $i=1, \cdots, n$.

(H2) If $x_{i}=0$, then $\mathcal{V}_{i}^{-}=0$. In particular, if $x \in X_{s}$, then $\mathcal{V}_{i}^{-}=0$ for $i=1, \cdots, m$.

(H3) $\mathcal{F}_{i}=0$ if $i>m$.

(H4) If $x \in X_{s}$, then $\mathcal{F}_{i}=0$ and $V_{i}^{+}=0$ for $i=1, \cdots, m$.

(H5) The pulses on the infected compartments must be uncoupled with the uninfected compartments; that is, $h(x(n T))$ is essentially $h(X(n T))$.

(H6) It holds that $h(0)=0$.

(H7) $r\left(Q^{q} \Phi_{M}(T)\right)<1$, where $\Phi_{M}(t)$ is the fundamental solution matrix of the system

$$
\frac{\mathrm{d} Z(t)}{\mathrm{d} t}=M(t) Z
$$

(H8) $r\left(P^{q} \Phi_{-V}(T)\right)<1$.

In the following, we study the threshold dynamics of system (2.1) and show that its basic reproduction number can be defined as the spectral radius of the so-called next infection operator as that in impulsive and periodic environment [16].

Let $Y(t, s), t \geq s$ be the evolution operator of the linear impulsive periodic system

$$
\left\{\begin{array}{l}
\frac{\mathrm{d} y(t)}{\mathrm{d} t}=-V(t) y, \quad t \neq n T, \\
y\left(t^{+}\right)=P y(t), \quad t=n T
\end{array}\right.
$$

where the explicit expression of $Y(t, s)$ can be found in [17], we omit it here. By assumption (H1)-(H8), we also know that the periodic solution of system (3.4) is asymptotically stable.

Now, we define the so-called next infection operator $L$ as follows:

$$
(L \phi)(t)=\lim _{a \rightarrow-\infty} \int_{a}^{t} Y(t, s) F(s) \phi(s) \mathrm{d} s, \quad \phi \in C_{\omega},
$$


where $C_{\omega}$ is defined as the ordered Banach space of all $\omega$-periodic functions from $R$ to $R^{m}$, equipped with the maximum norm $\|\cdot\|_{\infty}$, and the positive cone $C_{\omega}^{+} \triangleq\left\{\phi \in C_{\omega}: \phi(t) \geq 0, \forall t \in R\right\} ; \phi(s)$ is the initial distribution of infectious individuals.

The limit as a goes to infinity does exist, and the next infection operator $L$ is well defined, continuous, positive and compact on the domain. We now define the basic reproductive number as the spectral radius of $L$ is

$$
R_{0} \triangleq \rho(L) \text {. }
$$

From above discussion, we have the following results.

Lemma 3.3. Assume that (H1)-(H8) hold, Then the following statements are valid:

1) $R_{0}=1$ if and only if $r\left(P^{q} \Phi_{F-V}(\omega)\right)=1$.

2) $R_{0}>1$ if and only if $r\left(P^{q} \Phi_{F-V}(\omega)\right)>1$.

3) $R_{0}<1$ if and only if $r\left(P^{q} \Phi_{F-V}(\omega)\right)<1$.

The proof in detail is similar to periodic systems in [15].

Lemma 3.4. If $R_{0}<1$ the disease-free periodic solution $\tilde{x}(t)$ is asymptotically stable, and unstable if $R_{0}>1$.

Proof: Observe that the linearized system of system (3.3) at the disease-free periodic solution is

$$
\left\{\begin{array}{l}
\frac{\mathrm{d} x(t)}{\mathrm{d} t}=\left(\begin{array}{cc}
F(t)-V(t) & \mathbf{0} \\
-J(t) & M(t)
\end{array}\right) x(t), \quad t \neq k T, \\
x\left(t^{+}\right)=\left(\begin{array}{cc}
P & \mathbf{0} \\
\Gamma & Q
\end{array}\right) x(t), \quad t=k T .
\end{array}\right.
$$

Then the monodromy matrix of the impulsive system (3.5) equals

$$
\left(\begin{array}{ll}
P & 0 \\
\Gamma & Q
\end{array}\right)^{q}\left(\begin{array}{cc}
\Phi_{F-V}(T) & \mathbf{0} \\
* & \Phi_{M}(T)
\end{array}\right)
$$

where * represents a non-zero block matrix. Then the Floquet multipliers of system (3.3) are the eigenvalues of $r\left(P^{q} \Phi_{F-V}(T)\right)$ and $r\left(Q^{q} \Phi_{M}(T)\right)$. By assumption (H7), that is, $r\left(Q^{q} \Phi_{M}(T)\right)<1$, it then follows that the disease-free periodic solution is asymptomatically stable if $r\left(P^{q} \Phi_{F-V}(T)\right)<1$, and unstable if $r\left(P^{q} \Phi_{F-V}(T)\right)>1$. This completes the proof.

Following, we demonstrate the existence of the disease-free periodic solution. Set $I_{h}(t)=0, I_{v}(t)=0$ for all $t>0$. Under this condition, we have the following system:

$$
\left\{\begin{array}{l}
\frac{\mathrm{d} S_{h}(t)}{\mathrm{d} t}=-d_{1} S_{h}(t), \\
\frac{\mathrm{d} S_{v}(t)}{\mathrm{d} t}=\Lambda-d_{2} S_{v}(t), \\
S_{h}\left(t^{+}\right)=S_{h}(t)+\delta, \\
S_{v}\left(t^{+}\right)=S_{v}(t),
\end{array}\right\} t=k T,
$$

From the first and third equations of system (3.6), we have 


$$
\left\{\begin{array}{l}
\frac{\mathrm{d} S_{h}(t)}{\mathrm{d} t}=-d_{1} S_{h}(t), t \neq k T, \\
S_{h}\left(t^{+}\right)=S_{h}(t)+\delta, t=k T .
\end{array}\right.
$$

Then, over the $k$-th impulsive interval,

$S_{h}(t)=S_{h}\left(k T^{+}\right) \mathrm{e}^{-d_{1}(t-k T)}, t \in(k T,(k+1) T]$. By the impulsive condition, we have $S_{h}\left((k+1) T^{+}\right)=S_{h}\left(k T^{+}\right) \mathrm{e}^{-d_{1} T}+\sigma$. The unique fixed point of this system equals $S_{h}^{*}(t)=\frac{\sigma}{1-\mathrm{e}^{-d_{1} T}}$.

Accordingly, the impulsive periodic solution of the system (3.7) is

$$
S_{h}^{*}(t)=\frac{\sigma}{1-\mathrm{e}^{-d_{1} T}} \mathrm{e}^{-d_{1}(t-n T)}, \quad t \in(k T,(k+1) T] .
$$

Obviously, $S_{h}^{*}(t)$ is globally asymptomatically stable.

From system (3.6), we know that $S_{v}(t)$ is not affected by impulse, and we have $\lim _{t \rightarrow+\infty} S_{v}^{*}(t)=\Lambda d_{2}^{-1}$. Hence, system (2.1) has a unique disease-free periodic solution $E_{0}=\left(S_{h}^{*}(t), 0, S_{v}^{*}(t), 0\right)$.

Obviously, by Lemma 3.4, we have that $\left(S_{h}^{*}(t), 0, S_{v}^{*}(t), 0\right)$ of system (2.4) is asymptotically stable if $R_{0}<1$, and unstable if $R_{0}>1$.

We denote $x(t)=\left(I_{h}(t), I_{v}(t), S_{h}(t), S_{v}(t)\right)^{\mathrm{T}}$, then for system (2.1), we have

$$
\begin{gathered}
\mathcal{F}(t, x(t))=\left(\begin{array}{c}
\lambda S_{h}(t) I_{v}(t) \\
\beta S_{v}(t) I_{h}(t) \\
0 \\
0
\end{array}\right), \\
\mathcal{V}^{+}(t, x(t))=\left(\begin{array}{c}
0 \\
0 \\
0 \\
\Lambda
\end{array}\right), \quad \mathcal{V}^{-}(t, x(t))=\left(\begin{array}{c}
d_{1} I_{h}(t)+v I_{h}(t) \\
d_{2} I_{v}(t) \\
\lambda S_{h}(t) I_{v}(t)+d_{1} S_{h}(t) \\
\beta S_{v}(t) I_{h}(t)+d_{2} S_{v}(t)
\end{array}\right)
\end{gathered}
$$

and $\mathcal{V}(t, x(t))=\mathcal{V}^{-}(t, x(t))-\mathcal{V}^{+}(t, x(t))$

Furthermore, we denote $X=\left(I_{h}, I_{v}\right), Y=\left(S_{h}, S_{v}\right)$. By [15], suppose that $x(t)$ immediately after pulses equals

$$
x\left(t^{+}\right)=\psi(x(t)), \quad t=n T .
$$

For the system (2.1), we have

$$
\begin{aligned}
& \psi=(h, g)^{\mathrm{T}}, h=\left(\psi_{1}, \psi_{2}\right)=\left((1-\phi) I_{h}(t), I_{v}(t)\right), \\
& g=\left(\psi_{3}, \psi_{4}\right)=\left(S_{h}(t)+\delta, S_{v}(t)\right) .
\end{aligned}
$$

Clearly, conditions (H1)-(H6) are satisfied for system (2.1). There are only (H7) and (H8) should be verified in the following.

$x^{*}(t)=\left(0,0, S_{h}^{*}(t), S_{v}^{*}(t)\right)$ is the disease-free periodic solution for system (2.1). We define $f(t, x(t))=\mathcal{F}(t, x(t))-\mathcal{V}(t, x(t))$,

$M(t)=\left(\frac{\partial f_{i}\left(x^{*}(t)\right)}{\partial x_{j}}\right)_{3 \leq i, j \leq 4}$ and $Q=\left(\frac{\partial \psi_{i}\left(x^{*}(t)\right)}{\partial x_{j}}\right)_{3 \leq i, j \leq 4}$, where $f_{i}(x(t)), x_{i}$ 
and $\psi_{i}$ are the $i$-th component of $f(t, x(t)), x$ and $\psi$, respectively.

Then, from (3.8) and (3.9), we obtain

$$
M(t)=\left(\begin{array}{cc}
-d_{1} & 0 \\
0 & -d_{2}
\end{array}\right)
$$

From (3.10), we have

$$
Q=\left(\begin{array}{ll}
1 & 0 \\
0 & 1
\end{array}\right)
$$

and hence, $r\left(Q \Phi_{M}(T)\right)<1$. Therefore, (H7) holds.

We further denote $F(t), V(t)$ and $P$ are $2 \times 2$ matrices defined by $F(t)=\left(\frac{\partial \mathcal{F}_{i}\left(t, x^{*}(t)\right)}{\partial x_{j}}\right)_{1 \leq i, j \leq 2}, V(t)=\left(\frac{\partial \mathcal{V}_{i}\left(t, x^{*}(t)\right)}{\partial x_{j}}\right)_{1 \leq i, j \leq 2}$ and $P=\left(\frac{\partial \psi_{i}(x(t))}{\partial x_{j}}\right)_{1 \leq i, j \leq 2}$, where $\mathcal{F}_{i}(t, x)$ and $\mathcal{V}_{i}(t, x)$ are the $i$-th component of $\mathcal{F}(t, x)$ and $\mathcal{V}(t, x)$, respectively. Then from (3.8), (3.9) and (3.10), it follows that

$$
F(t)=\left(\begin{array}{cc}
0 & \lambda S_{h}^{*}(t) \\
\beta S_{v}^{*}(t) & 0
\end{array}\right), V(t)=\left(\begin{array}{cc}
d_{1}+v & 0 \\
0 & d_{2}
\end{array}\right) \text { and } P=\left(\begin{array}{cc}
1-\phi & 0 \\
0 & 1
\end{array}\right) .
$$

It is easy to see that $r\left(P^{q} \Phi_{-V}(\omega)\right)<1$ satisfied. (H8) is hold.

Thus, the Lemma 3.3 is right for system (2.1).

\section{Global Stability of the Disease-Free Equilibrium}

In this section, we prove that the disease-free periodic solution $E_{0}=\left(S_{h}^{*}(t), 0, S_{v}^{*}(t), 0\right)$ is globally asymptotically stable, if $R_{0}<1$ and hence, the disease extinct.

Firstly, we need to prove the following lemma.

Lemma 4.1. For the system (2.1), it holds that

$$
\lim _{t \rightarrow+\infty}\left(N_{h}(t)-S_{h}^{*}(t)\right)=0, \quad \lim _{t \rightarrow+\infty}\left(N_{v}(t)-S_{v}^{*}(t)\right)=0,
$$

where

$$
N_{h}(t)=S_{h}(t)+I_{h}(t), N_{v}(t)=S_{v}(t)+I_{v}(t) .
$$

Proof: Let $y_{1}(t)=N_{h}(t)-S_{h}^{*}(t)$, from Theorem 2.1, we have

$$
\begin{aligned}
\frac{\mathrm{d} y_{1}(t)}{\mathrm{d} t} & =\frac{\mathrm{d} N_{h}(t)}{\mathrm{d} t}-\frac{\mathrm{d} S_{h}^{*}(t)}{\mathrm{d} t}=-d_{1} N_{h}(t)-v N_{h}(t)-d_{1} S_{h}^{*}(t) \\
& \leq-d_{1}\left(N_{h}(t)-S_{h}^{*}(t)\right)=-d_{1} y(t),
\end{aligned}
$$

and

$$
\begin{aligned}
y_{1}\left(t^{+}\right) & =N_{h}\left(t^{-}\right)-S_{h}^{*}\left(t^{+}\right) \\
& =N_{h}(t)-\psi I_{h}(t)+\delta-S_{h}^{*}(t)-\delta \\
& \leq N_{h}(t)-S_{h}^{*}(t)=y_{1}(t),
\end{aligned}
$$


for $t_{2}>t_{1}$.

Obviously, by (4.1), (4.2) and the comparison principle of impulsive differential equations in [17], we have

$$
\lim _{t \rightarrow+\infty} y_{1}(t)=\lim _{t \rightarrow+\infty}\left(N_{h}(t)-S_{h}^{*}(t)\right)=0 .
$$

In similar method, we can prove

$$
\lim _{t \rightarrow+\infty}\left(N_{v}(t)-S_{v}^{*}(t)\right)=0
$$

for $t_{2}>t_{1}$.

Hence, the proof is completed.

Theorem 4.1. For any solution of system (2.1), if $R_{0}<1$, then the disease-free periodic solution $\left(S_{h}^{*}(t), 0, S_{v}^{*}(t), 0\right)$ is globally asymptotically stable and if $R_{0}>1$, then it is unstable.

Proof: By Lemma 3.3, if $R_{0}>1$, then $\left(S_{h}^{*}(t), 0, S_{v}^{*}(t), 0\right)$ is unstable and if $R_{0}<1$, then $\left(S_{h}^{*}(t), 0, S_{v}^{*}(t), 0\right)$ is locally stable. Hence, it is sufficient to show that the global attractivity of $\left(S_{h}^{*}(t), 0, S_{v}^{*}(t), 0\right)$ for $R_{0}<1$.

Now, we prove the global attractivity of the disease-free solution.

From Lemma 4.1, there exist a $t_{3} \geq t_{2}$ and a positive constant $\epsilon_{1}$ such that $S_{h}(t) \leq S_{h}^{*}(t)+\epsilon_{1}, \quad S_{v}(t) \leq S_{v}^{*}(t)+\epsilon_{1}$.

By the second, fourth, sixth and eighth equations of system (2.1), we have

$$
\left\{\begin{array}{l}
\frac{\mathrm{d} I_{h}(t)}{\mathrm{d} t} \leq \lambda\left(S_{h}^{*}(t)+\epsilon_{1}\right) I_{v}(t)-\left(d_{1}+v\right) I_{h}(t), \\
\frac{\mathrm{d} I_{v}(t)}{\mathrm{d} t} \leq \beta\left(S_{v}^{*}(t)+\epsilon_{1}\right) I_{h}(t)-d_{2} I_{v}(t), \\
I_{h}\left(t^{+}\right)=(1-\phi) I_{h}(t), \\
I_{v}\left(t^{+}\right)=I_{v}(t),
\end{array}\right\} t=k T,
$$

for $t \geq t_{3}$.

Set $M_{\epsilon_{1}}(t)$ be the $2 \times 2$ matrix function such that

$$
M_{\epsilon_{1}}(t)=\left(\begin{array}{cc}
0 & \epsilon_{1} \\
\epsilon_{1} & 0
\end{array}\right) \text {. }
$$

By Lemma 3.3, we have $r\left(P^{q} \Phi_{F-V}(\omega)\right)<1$, we restrict $\epsilon_{1}>0$, such that $r\left(P^{q} \Phi_{F-V+M_{\mathrm{q}}}(\omega)\right)<1$. Let us consider the following system

$$
\left\{\begin{array}{l}
\frac{\mathrm{d} I_{h}(t)}{\mathrm{d} t}=\lambda\left(S_{h}^{*}(t)+\epsilon_{1}\right) I_{v}(t)-\left(d_{1}+v\right) I_{h}(t), \\
\frac{\mathrm{d} I_{v}(t)}{\mathrm{d} t}=\beta\left(S_{v}^{*}(t)+\epsilon_{1}\right) I_{h}(t)-d_{2} I_{v}(t), \\
I_{h}\left(t^{+}\right)=(1-\phi) I_{h}(t), \\
I_{v}\left(t^{+}\right)=I_{v}(t),
\end{array}\right\} t=k T, \quad t=k T .
$$

By Lemma 3.1 and the standard comparison principle, there exists a positive $T$-periodic function $V(t)=\left(V_{1}(t), V_{2}(t)\right)$ such that $J_{1}(t) \leq V(t) \exp \left(p_{1} t\right)$ 
where $J_{1}(t)=\left(I_{h}(t), I_{v}(t)\right)^{\mathrm{T}}$ and $p_{1}=\frac{1}{T} \ln \left(r\left(P^{q} \Phi_{F-V+M_{\mathrm{e}}}\right)(T)\right)<0$. Then, we see that $\lim _{t \rightarrow \infty} I_{h}(t)=0$ and $\lim _{t \rightarrow \infty} I_{v}(t)=0$.

Moreover, we obtain that $\lim _{t \rightarrow \infty}\left(S_{h}(t)-S_{h}^{*}(t)\right)=0, \lim _{t \rightarrow \infty}\left(S_{v}(t)-S_{v}^{*}(t)\right)=0$. Hence, the disease-free periodic solution $\left(S_{h}^{*}(t), 0, S_{v}^{*}(t), 0\right)$ is globally attractive. This completes the proof.

\section{Permanence}

In this section, we show that if $R_{0}>1$, then the disease persists.

Let $\tilde{X}$ be a matrix space, $f: \tilde{X} \rightarrow \tilde{X}$ be a continuous map, and $X_{0} \subseteq \tilde{X}$ be an open set. Define

$$
\partial X_{0}:=\tilde{X} \backslash X_{0}, M_{\partial}:=\left\{x \in \partial X_{0}: f^{n}(x) \in \partial X_{0}, n \geq 0\right\} .
$$

$A_{\partial}$ is a maximal compact invariant set of $f$ in $\partial X_{0}$. A finite sequence $\left\{M_{1}, \cdots, M_{k}\right\}$ are disjoint, compact, and invariant subsets of $\partial X_{0}$, and each of them is isolated in $\partial X_{0}$.

We present persistence theory [18] as follows:

Lemma 5.1. Assume that

1) $f\left(X_{0}\right) \subset X_{0}$ and $f$ has a global attractor $A$;

2) The maximal compact invariant set $A_{\partial}=A \cap M_{\partial}$ of $f$ in $\partial X_{0}$, possibly empty, has an acyclic covering $\tilde{M}$ and where $\tilde{M}=\left\{M_{1} \cdots, M_{k}\right\}$ with the following properties:

a) $M_{i}$ is isolated in $\tilde{X}$; b) $W^{s}\left(M_{i}\right) \cap X_{0}=\varnothing$ for each $1 \leq i \leq k$.

Then, $f$ is uniformly persistent with respect to $\left(X_{0}, \partial X_{0}\right)$, i.e., there is $\eta>0$ such that for any compact internally chain transitive set $L$ with $L \nsubseteq M_{i}$ for all $1 \leq i \leq k, \inf _{x \in L} d\left(x, \partial X_{0}\right)>\eta$.

Define Poincaré map $\tilde{P}: R_{+}^{4} \rightarrow R_{+}^{4}$ associated with system (2.1), satisfying $\tilde{P}\left(x^{0}\right)=u\left(\omega^{+}, X^{0}\right), \forall x^{0} \in R_{+}^{4}$, where $u\left(t, x^{0}\right)$ is the unique solution of system (2.1) with $u\left(0, x^{0}\right)=x^{0}$. Now, we denote $\tilde{X}=\left\{\left(S_{h}, I_{h}, S_{v}, I_{v}\right) \in R_{+}^{4}\right\}$, $X_{0}=\left\{\left(S_{h}, I_{h}, S_{v}, I_{v}\right) \in\left\{S_{h} \geq 0, I_{h}>0, S_{v} \geq 0, I_{v}>0\right\}\right.$ and $\partial X_{0}=\tilde{X} \backslash X_{0}$.

Theorem 5.1. Suppose that $R_{0}>1$, then system (2.1) exists a positive constant $\epsilon>0$ such that for all $\left(S_{h}(0), I_{h}(0), S_{v}(0), I_{v}(0)\right)=\left(S_{h}^{0}, I_{h}^{0}, S_{v}^{0}, I_{v}^{0}\right) \in X_{0}$,

$$
\liminf _{t \rightarrow+\infty} I_{h}(t) \geq \epsilon, \quad \liminf _{t \rightarrow+\infty} I_{v}(t) \geq \epsilon .
$$

Proof: Firstly, we prove that $\tilde{P}$ is uniformly persistent with respect to $\left(X_{0}, \partial X_{0}\right)$. From Theorem 2.1, it is obvious that $\tilde{X}$ and $X_{0}$ are positively invariant. We also know that $\tilde{P}$ is point dissipative on $R_{+}^{4}$ from Lemma 4.1.

Denote $M_{\partial}=\left\{\left(S_{h}^{0}, I_{h}^{0}, S_{v}^{0}, I_{v}^{0}\right) \in \partial X_{0} \mid \tilde{P}^{m}\left(S_{h}^{0}, I_{h}^{0}, S_{v}^{0}, I_{v}^{0}\right) \in \partial X_{0}, \forall m \geq 0\right\}$.

Next, we need to show that $M_{\partial}=\left\{\left(S_{h}, 0, S_{v}, 0\right) \mid S_{h} \geq 0, S_{v} \geq 0\right\}$.

Obviously, $\left\{\left(S_{h}, 0, S_{v}, 0\right) \mid S_{h} \geq 0, S_{v} \geq 0\right\} \subseteq M_{\partial}$. We now need to prove that $M_{\partial} \backslash\left\{\left(S_{h}, 0, S_{v}, 0\right) \mid S_{h} \geq 0, S_{v} \geq 0\right\}=\varnothing$. Suppose it's not hold. For any $\left(S_{h}^{0}, I_{h}^{0}, S_{v}^{0}, I_{v}^{0}\right) \in M_{\partial} \backslash\left\{\left(S_{h}, 0, S_{v}, 0\right), S_{h} \geq 0, S_{v} \geq 0\right\}=\varnothing \quad$. For the case $I_{h}^{0}=0, I_{v}^{0}>0$, it is obvious that $I_{v}(t)>0$ and $S_{h}(t)>0$ for all $t>0$. From second and sixth equations of system (2.4), we have 


$$
\left\{\begin{array}{l}
\frac{\mathrm{d} I_{h}(t)}{\mathrm{d} t}=\lambda S_{h}(t) I_{v}(t)-\left(d_{1}+v\right) I_{h}(t), \quad t \neq k T \\
I_{h}\left(t^{+}\right)=(1-\phi) I_{h}(t), \quad t=k T
\end{array}\right.
$$

then it hold that $I_{h}(t)=\frac{\lambda S_{h}(0) I_{h}(0)}{d_{1}+v}+\left(I_{h}^{*}-\frac{\lambda S_{h}(0) I_{h}(0)}{d_{1}+v}\right) \mathrm{e}^{-\left(d_{1}+v\right)(t-T)}>0$ for all $t>0$ from Lemma 3.2, where $I_{h}^{*}=\frac{\lambda S_{h}(0) I_{h}(0)}{d_{1}+v} \frac{(1-\omega)\left(1-\mathrm{e}^{-\left(d_{1}+v\right) T}\right)}{1-(1-\omega) \mathrm{e}^{-\left(d_{1}+v\right) T}}$. In the similar method, for the case $I_{h}^{0}>0, I_{v}^{0}=0$, then we have $I_{h}(t)>0$ and $S_{v}(t)>0$ for all $t>0$. This implies that $\left(S_{h}, I_{h}, S_{v}, I_{v}\right) \notin M_{\partial}$ for $t(>0)$ sufficiently small. It follows that $M_{\partial} \subseteq\left\{\left(S_{h}, 0, S_{v}, 0\right) \mid S_{h} \geq 0, S_{v} \geq 0\right\}$. Thus, $M_{\partial}=\left\{\left(S_{h}, 0, S_{v}, 0\right) \mid S_{h} \geq 0, S_{v} \geq 0\right\}$. It is clear that $E_{0}=\left(S_{h}^{*}, 0, S_{v}^{*}, 0\right)$ is a unique fixed point of $\tilde{P}$ in $M_{\partial}$.

In the following, we need to prove $W^{s}\left(E_{0}\right) \cap X_{0}=\varnothing$.

We write $x^{0}=\left(S_{h}^{0}, I_{h}^{0}, S_{v}^{0}, I_{v}^{0}\right) \in X_{0}$. By the continuity of the solutions with respect to the initial conditions, $\forall \epsilon>0$, there exist $\delta_{0}>0$, such that for all $x^{0} \in X_{0}$ with $x^{0}-E_{0} \leq \delta_{0}$, it hold that

$$
\left\|u\left(t, x^{0}\right)-u\left(t, E_{0}\right)\right\| \leq \varepsilon, \quad \forall t \in[0, T] .
$$

Now, we show that

$$
\limsup _{t \rightarrow \infty} d\left(\tilde{P}^{m}\left(x^{0}\right), E_{0}\right) \geq \delta_{0} .
$$

Suppose not hold, then $\limsup d\left(\tilde{P}^{m}\left(x^{0}\right), E_{0}\right)<\delta_{0}$ for some $x^{0} \in X_{0}$. Without loss of the generality, we can assume that $d\left(\tilde{P}^{m}\left(x^{0}\right), E_{0}\right)<\delta_{0}, \forall m \geq 0$. Thus, we obtain that $\left\|u\left(t, \tilde{P}^{m}\left(x^{0}\right)\right)-u\left(t, E_{0}\right)\right\| \leq \varepsilon, \forall t \in[0, T]$ and $\forall m \geq 0$.

For any $\tilde{t}_{1} \geq 0$, let $\tilde{t}_{1}=m \omega+t^{\prime}$, where $t^{\prime} \in[0, \omega]$ and $m=\left[\frac{t_{1}}{\omega}\right] \cdot\left[\frac{t_{1}}{\omega}\right]$ is the greatest integer less than or equal to $t_{1} / \omega$. So, we have that

$$
\left\|u\left(\tilde{t}_{1}, x^{0}\right)-u\left(\tilde{t}_{1}, E_{0}\right)\right\|=\left\|u\left(t^{\prime}, \tilde{P}^{m}\left(x^{0}\right)\right)-u\left(t^{\prime}, E_{0}\right)\right\| \leq \varepsilon, \forall \tilde{t}_{1} \geq 0 .
$$

It follows that

$$
0 \leq I_{h}(t) \leq \epsilon, 0 \leq I_{v}(t) \leq \varepsilon, \forall \tilde{t}_{2}>\tilde{t}_{1} .
$$

Then, by the first, third, fifth and seventh equations of system (2.1), we have

$$
\left\{\begin{array}{l}
\frac{\mathrm{d} S_{h}(t)}{\mathrm{d} t} \geq-\left(\lambda \varepsilon+d_{1}\right) S_{h}(t), \\
\frac{\mathrm{d} S_{v}(t)}{\mathrm{d} t} \geq \Lambda-\beta \varepsilon S_{v}(t)-d_{2} S_{v}(t), \\
S_{h}\left(t^{+}\right)=S_{h}(t)+\delta, \\
S_{v}\left(t^{+}\right)=S_{v}(t),
\end{array}\right\} t=k T,
$$

Consider an auxiliary system 


$$
\left\{\begin{array}{l}
\frac{\mathrm{d} \tilde{S}_{h}(t)}{\mathrm{d} t} \geq-\left(\lambda \varepsilon+d_{1}\right) \tilde{S}_{h}(t), \\
\frac{\mathrm{d} \tilde{S}_{v}(t)}{\mathrm{d} t} \geq \Lambda-\beta \varepsilon \tilde{S}_{v}(t)-d_{2} \tilde{S}_{v}(t), \\
\tilde{S}_{h}\left(t^{+}\right)=\tilde{S}_{h}(t)+\delta, \\
\tilde{S}_{v}\left(t^{+}\right)=\tilde{S}_{v}(t),
\end{array}\right\} t \neq k T
$$

Using the same method as aforementioned, we have that (5.3) admits a positive periodic solution $\left(\tilde{S}_{h}^{*}(t), \tilde{S}_{v}^{*}(t)\right)$. Since $r\left(Q^{q} \Phi_{M}(t)\right)<1$ holds. Then, there exists a small enough $\varepsilon$ such that $r\left(Q^{q} \Phi_{M+M_{\varepsilon}}(t)\right)<1$, and $r\left(Q^{q} \Phi_{M+M_{\varepsilon}}(t)\right)$ is continuous for small $\varepsilon$, where

$$
M_{\varepsilon}=\left(\begin{array}{cc}
\varepsilon & 0 \\
0 & \varepsilon
\end{array}\right) \text {. }
$$

As before, we have that $\left(\tilde{S}_{h}^{*}(t), \tilde{S}_{v}^{*}(t)\right)$ is globally asymptotically stable, and meanwhile $\lim _{\varepsilon \rightarrow 0} \tilde{S}_{h}^{*}(t)=S_{h}^{*}(t), \lim _{\varepsilon \rightarrow 0} \tilde{S}_{v}^{*}(t)=S_{v}^{*}(t)$, thus there exist $\varepsilon_{1}$ small enough and a constant $\xi>0$, such that

$$
\left\{\begin{array}{l}
\tilde{S}_{h}^{*}(t) \geq S_{h}^{*}(t)-\xi, \\
\tilde{S}_{v}^{*}(t) \geq S_{v}^{*}(t)-\xi
\end{array}\right.
$$

for $\xi<\varepsilon_{1}$.

On the other hand, the standard comparison theorem implies that there exist $\tilde{t}_{2} \geq \tilde{t}_{1}$ and $\xi_{1}$ such that

$$
\left\{\begin{array}{l}
S_{h}(t) \geq \tilde{S}_{h}(t) \geq \tilde{S}_{h}^{*}(t)-\xi_{1}, \\
S_{v}(t) \geq \tilde{S}_{v}(t) \geq \tilde{S}_{v}^{*}(t)-\xi_{1},
\end{array}\right.
$$

for all $\tilde{t}_{3} \geq \tilde{t}_{2}$. Then, for all $\tilde{t}_{3} \geq \tilde{t}_{2}$, we have

$$
\left\{\begin{array}{l}
S_{h}(t) \geq S_{h}^{*}(t)-\xi-\xi_{1} \triangleq S_{h}^{*}(t)-\gamma, \\
S_{v}(t) \geq S_{v}^{*}(t)-\xi-\xi_{1} \triangleq S_{v}^{*}(t)-\gamma,
\end{array} \quad \forall t \geq t_{2}\right.
$$

where $\gamma=\xi+\xi_{1}$.

By the second, fourth, sixth and eighth equations of system (2.1), we have

$$
\left\{\begin{array}{l}
\frac{\mathrm{d} I_{h}(t)}{\mathrm{d} t} \geq \lambda\left(S_{h}^{*}(t)-\gamma\right) I_{v}(t)-\left(d_{1}+v\right) I_{h}(t), \\
\frac{\mathrm{d} I_{v}(t)}{\mathrm{d} t} \geq \beta\left(S_{v}^{*}(t)-\gamma\right) I_{h}(t)-d_{2} I_{v}(t), \\
I_{h}\left(t^{+}\right)=(1-\phi) I_{h}(t), \\
I_{v}\left(t^{+}\right)=I_{v}(t),
\end{array}\right\} t \neq k T,
$$

Set $M_{\gamma}$ be the $2 \times 2$ matrix function such that

$$
M_{\gamma}=\left(\begin{array}{ll}
0 & \gamma \\
\gamma & 0
\end{array}\right)
$$

where $\gamma$ is small enough. 
By Lemma 3.1 and the standard comparison principle, it follows that there exists a positive $T$-periodic function $W(t)=\left(W_{1}(t), W_{2}(t)\right)$ such that $J_{2}(t)=\exp \left(p_{2} t\right) W(t)$ is a solution of system (5.4), where $p_{2}=\frac{1}{T} \ln r\left(P^{q} \Phi_{F-V-M_{\gamma}}(T)\right)$. Since $r\left(P^{q} \Phi_{F-V}(T)\right)>1$, and $r\left(P^{q} \Phi_{F-V-M_{\gamma}}(T)\right)$ is continuous for small $\gamma$. So we can choose $\gamma$ small enough, such that $r\left(P^{q} \Phi_{F-V-M_{\gamma}}(T)\right)>1$. It follows that $p_{2}>0$, we can choose $\tilde{t}_{4} \geq \tilde{t}_{3}$ such that

$$
I_{h}\left(\tilde{t}_{4}\right) \geq W_{1}(0), I_{v}\left(\tilde{t}_{4}\right) \geq W_{2}(0) .
$$

By the comparison principle we have

$$
I_{h}(t) \geq \exp ^{p_{2}\left(t-\tilde{t}_{4}\right)} W_{1}\left(t-\tilde{t}_{4}\right), I_{v}(t) \geq \exp ^{p_{2}\left(t-\tilde{t}_{4}\right)} W_{2}\left(t-\tilde{t}_{4}\right)
$$

for all $t \geq \tilde{t}_{4}$. Then, we obtain that $\lim _{t \rightarrow+\infty} I_{h}(t)=+\infty$ and $\lim _{t \rightarrow+\infty} I_{v}(t)=+\infty$, which contradicts to the boundedness of $0 \leq I_{h}(t) \leq \varepsilon, \quad 0 \leq I_{h}(\vec{t}) \leq \varepsilon$. Thus we have proved $W^{s}\left(E_{0}\right) \cap X_{0}=\varnothing$, which implies each orbit in $M_{\partial}$ converges to $E_{0}$, and hence $E_{0}$ is acyclic in $M_{\partial}$.

Therefore, the Lemma 5.1 is satisfied for system (2.1). Furthermore, we obtain that the disease is permanence, when $R_{0}>1$.

\section{Conclusion}

In this paper, a vector-borne epidemic model for Huanglongbing with impulsive control is established. Under the reasonable assumptions (H1)-(H8), one studied the threshold dynamics behavior of the model. Based on comparison theorem of impulsive differential equation and method of enlarging and reducing, we proved that if the $R_{0}<1$, the disease-free equilibrium is global stability, and Huanglongbing is uniformly persistent if $R_{0}>1$. We only consider replanting susceptible and rouging infective in model, spraying insecticides to kill psyllid is not. It's a lot of room for us to improve.

\section{Acknowledgements}

The research has been supported by the Science and Technology Plan Projects of Jiangxi Provincial Education Department (GJJ151491, GJJ171373), Guidance Project of Ji'an Science and Technology Bureau, the Natural Science Foundation of Ji'an College (16JY103).

\section{Conflicts of Interest}

The authors declare no conflicts of interest regarding the publication of this paper.

\section{References}

[1] Bové, J.M. (2006) Huanglongbing: A Destructive, Newly Emerging, Century-Old Disease of Citrus. Journal of Plant Pathology, 88, 7-37. 
[2] Vilamiu, R.G.A., Ternes, S., et al. (2012) A Model for Huanglongbing Spread between Citrus Plants Including Delay Times and Human Intervention. https://doi.org/10.1063/1.4756657

[3] Gao, S.J., Luo, L., Yan, S.X., et al. (2018) Dynamical Behavior of a Novel Impulsive Switching Model for HLB with Seasonal Fluctuations. Complexity, 2018, Article ID: 2953623. https://doi.org/10.1155/2018/2953623

[4] Luo, L., Gao, S.J., et al. (2017) Transmission Dynamics of a Huanglongbing Model with Cross Protection. Advances in Difference Equations, 2017, 355. https://doi.org/10.1186/s13662-017-1392-y

[5] Van der Plank, J.E. (1963) Plant Diseases: Epidemics and Control. Academic Press, New York. https://doi.org/10.1097/00010694-196410000-00018

[6] Kranz, J. (1974) The Role and Scope of Mathematical Analysis and Modeling in Epidemiology. In: Kranz, J., Ed., Epidemics of Plant Diseases; Mathematical Analysis and Modeling, Springer, Berlin, Heidelberg, New York, 7-54. https://doi.org/10.1007/978-3-642-96220-2_2

[7] Chiyaka, C., Singer, B.H., Halbert, S.E., Morris, J.G. and van Bruggen, A.H.C. (2012) Modeling Huanglongbing Transmission within a Citrus Tree. Proceedings of the National Academy of Sciences of the United States of America, 109, 12213-12218. https://doi.org/10.1073/pnas.1208326109

[8] Wang, J.P., Gao, S.J., et al. (2014) Threshold Dynamics of a Huanglongbing Model with Logistic Growth in Periodic Environments. Abstract and Applied Analysis, 2014, Article ID: 841367. https://doi.org/10.1155/2014/841367

[9] Fishman, S. and Marcus, R. (1984) A Model for Spread of Plant Disease with Periodic Removals. Journal of Mathematical Biology, 21, 149-158. https://doi.org/10.1007/BF00277667

[10] Jeger, M.J., Holt, J., van den Bosch, F. and Madden, L.V. (2004) Epidemiology of Insect-Transmitted Plant Viruses: Modelling Disease Dynamics and Control Interventions. Physiological Entomology, 29, 291-304. https://doi.org/10.1111/j.0307-6962.2004.00394.x

[11] Zhang, X.S. and Holt, J. (2004) Mathematical Models of Cross Protection in the Epidemiology of Plant-Virus Diseases. Phytopathology, 91, 924-934. https://doi.org/10.1094/PHYTO.2001.91.10.924

[12] Tang, S.Y., Xiao, Y.N. and Cheke, R.A. (2010) Dynamical Analysis of Plant Disease Models with Cultural Control Strategies and Economic Thresholds. Mathematics and Computers in Simulation, 80, 894-921. https://doi.org/10.1016/j.matcom.2009.10.004

[13] Ayres, A.J., Massari, C.A., Lopes, S.A., et al. (2005) Proceedings of 2nd International Citrus Canker and Huanglongbing Research Workshop, Florida Citrus Mutual, Orlando, $\mathrm{H}-4$.

[14] Gao, S.J., Tu, Y.B. and Wang, J.L. (2018) Basic Reproductive Number for a General Hybrid Epidemic Model. Advances in Difference Equations, 2018, 310. https://doi.org/10.1186/s13662-018-1707-7

[15] Yang, Y.P. and Xiao, Y.N. (2012) Threshold Dynamics for Compartmental Epidemic Models with Impulses. Nonlinear Analysis: Real World Applications, 13, 224-234. https://doi.org/10.1016/j.nonrwa.2011.07.028

[16] Wang, W. and Zhao, X.-Q. (2008) Threshold Dynamics for Compartmental Epidemic Models in Periodic Environments. Journal of Dynamics and Differential Equations, 20, 699-717. https://doi.org/10.1007/s10884-008-9111-8 
[17] Lakshmikantham, V., Bainov, D.D. and Simeonov, P.S. (1989) Theory of Impulsive Differential Equations. World Scientific, Singapore. https://doi.org/10.1142/0906

[18] Zhao, X.-Q. (2003) Dynamical System in Population Biology. Springer-Verlag, New York. 